\title{
Non-fatal repetition of self-harm: population-based prospective cohort study in Taiwan
}

\author{
Vincent C. H. Chen, Happy K. L. Tan, Andrew T. A. Cheng, Chung-Ying Chen, Long-Ren Liao, \\ Robert Stewart, Michael Dewey and Martin Prince
}

\section{Background}

Repeated self-harm is relatively common and is linked with an elevated risk of eventual suicide. There has been no study of this involving a large sample from the Far East.

\begin{abstract}
Aims
To estimate the risk over the medium term of non-fatal repetition of self-harm and identify predictive factors in those carrying out self-harm.
\end{abstract}

\section{Method}

A total of 970 individuals who had self-harmed were recruited from a community-based suicide behaviour register system in Nantou, Taiwan from July 2000 to February 2003. Information regarding demography and suicide methods was collected. Individuals were followed-up until December 2005 to examine the risk of repeated self-harm and independent predictive factors

\section{Results}

Ninety cohort members had repeated self-harm during the follow-up period (accounting for 131 repeated self-harm episodes in all). The cumulative risks were $5.7 \%$ for the first year, $7.8 \%$ for the second year and $9.5 \%$ for the fourth year. The risk was highest within the first year after the self-harm event. Independent risk factors included female gender and self-cutting as well as self-poisoning with drugs. Effect of younger age was mediated through the choice of methods.

\section{Conclusions}

Individuals with self-harm have a high risk of repetition, especially within the first year. Suicide prevention strategies need to focus on intervening with this population to reduce their repetition.

\section{Declaration of interest}

None.
Approximately $15 \%$ of those who self-harm will do so again within a year, and the cumulative risk continues to increase to $23 \%$ after more than 4 years. ${ }^{1}$ Nearly half of the people seen in emergency departments following self-harm have a history of previous self-harm. ${ }^{2}$ Repetition increases the risk of future suicide, ${ }^{3}$ especially for young women. ${ }^{4}$ Therefore, from both the public health and clinical aspects, it is important to get more information about repetition of self-harm. However, according to the finding of one systemic review, there are few studies that have followed up the repetition of self-harm over more than 2 years, and no studies with a large sample from the Far East area.

In Taiwan, suicide is the ninth leading cause of mortality. Suicide rates have increased more than three-fold during the past 10 years. From July 2000 to December 2005, a community-based suicide register system was set up in Nantou County in Taiwan, which provided us with the opportunity, for the first time, to chart accurately the incidence of suicide and self-harm in the community. Using data from the register, we describe the characteristics of approximately 1000 episodes of self-harm and trace the risk of non-fatal repetition during a 5.5 year period, examining the relationship between the incidence of repetition and demographic factors as well as suicide method.

\section{Method}

\section{Participants and procedure}

All participants resided in Nantou County, Mid Taiwan, close to the centre of a major earthquake on September 21, 1999. With the support of government, a community mental health centre was established to provide mental health services for survivors. The centre comprised 20 staff including psychiatric nurses, psychologists and social workers, supervised by two senior psychiatrists. A new reporting system (Nantou suicide register) was established in June 2000 to identify all inhabitants who attempted suicide, using all available key informants and referral sources in the community. Through the Nantou suicide register, all Nantou residents who had carried out self-harm were recorded from July 2000 to February 2003 and then followed up through to December 2005. Although 1083 episodes of self-harm were recorded, we excluded those who did not live in Nantou county because we could not be sure whether any repeated selfharm acts would be ascertained with our register system. In this study we included any episodes of self-harm referred by all kinds of community resources. The first episode of self-harm during the study period was defined as the index episode.

A structured data coding form was designed to collect basic sociodemographic information (age, gender, education, marital status, address) and information regarding the act (date of the self-harm and methods used). This form was completed for all individuals who self-harmed by a group of informants working in all agencies and settings where self-harm was routinely identified. A meeting was held every 3 months with leaders of all referral sources to review the operation of the reporting system. The Nantou Health Bureau officers monitored the health sector regularly. All completed forms were forwarded to the community mental health centre within 24 hours. Staff at the centre checked the forms to identify any missing or unclear information, which was then completed during their weekly visits to the agencies/ settings via further enquiry with informants. This study was approved by the Tsaotun Psychiatric Center Research Ethics Committee.

\section{Statistical analysis}

All index self-harm episodes were followed up through the suicide register for any further acts of self-harm until December 2005. We describe first the demographic characteristics and methods used for individuals with self-harm. We calculated both cumulative and annual risks of repetition of self-harm across the 5 years of 
follow-up. Annual risks were calculated based on individuals (for which purpose only the first repeated self-harm was counted, and individuals could contribute to the numerator in only one annual follow-up period) and on episodes (for which purpose, any repeated self-harm was counted, and an individual could contribute to the numerator in more than one annual follow-up period). Regarding the analysis of risk factors for repeated selfharm, previous studies had often used time to the first repeat as the outcome measure. ${ }^{5,6}$, This ignores the fact that some individuals will repeat self-harm on multiple occasions, producing a count outcome variable $(0,1,2,3$ etc. acts of repeated selfharm). Given that it was likely that those with more episodes may be more likely to self-harm again, ${ }^{7}$ these count data were unlikely to conform strictly to a Poisson distribution. We decided therefore to explore whether negative binomial regression, recommended for these circumstances, ${ }^{8}$ might be a more appropriate analytical paradigm. The resulting regression models would be used to estimate an incidence rate ratio for non-fatal repetition according to gender, age, education, marital status and method used at index episode.

We also adjusted for the lengths of follow-up time, which varied between participants, by adding the log of follow-up time in months as a covariate. Risk ratios with $95 \%$ confidence intervals are presented. Analyses were carried out using STATA for Windows version 9.0.

\section{Results}

\section{Characteristics of participants}

The characteristics of the self-harm cohort are shown in Table 1. In total 970 people from Nantou County carried out an index act of self-harm and were recruited through the Nantou suicide register system from July 2000 to February 2003. Their ages ranged from 11 to 90 years (mean 37.2, s.d. $=15.9$ ). The gender ratio (female:male) was 1.7:1. Most were married, and more than two-thirds had fewer than 10 years of education. The follow-up duration ranged from 1 day to 5.5 years (mean 3.7, s.d.=1.5). Overall, the most commonly used suicide methods were selfpoisoning with drugs or other chemicals (44\%), self-poisoning with pesticides $(24 \%)$ and self-cutting $(23 \%)$. None used firearms. The most common method used for males was pesticide ingestion $(41.2 \%)$ but females tended to choose drugs or other chemicals $(51.8 \%)$. The difference in methods employed by males and females is significant $(P<0.001)$. For age, the proportion of those using pesticides increases with increasing age; whereas younger people are more likely to cut themselves. Differences in methods employed by age are also significant $(P<0.001)$.

\section{Risk of repeated self-harm and related factors}

Overall, 90 of the 970 individuals who self-harmed (9.3\%) carried out further acts of self-harm during the 5.5-year follow-up, of whom 67 (74\%) repeated once, $14(16 \%)$ twice, and $9(10 \%)$ three or more times. The mean number of repetitions in the sample was 1.2 with a standard deviation of 0.2 . Out of the 970 individuals, $10.8 \%$ of females and $6.7 \%$ of males harmed themselves again. The annual risk of non-fatal repetition based on person (risk of first non-fatal repetition) indicated that $5.7 \%$ of participants harmed themselves again in the first year, and a further $2.2 \%$ in the second year, $0.9 \%$ in the third year, and $0.9 \%$ in the fourth year. The cumulative risks of first non-fatal repetition were therefore $5.7 \%$ by the end of the first year, $7.8 \%$ by the end of the second year and $9.5 \%$ by the end of the fourth year (Table 2). The annual risks of non-fatal repetition based on episode (risk of any non-fatal repetition) were $5.7 \%$ in the first

\begin{tabular}{|c|c|c|}
\hline Characteristics & Self-harm, $n$ & $\%$ (valid) $^{a}$ \\
\hline \multicolumn{3}{|l|}{ Gender } \\
\hline Female & 611 & 63.0 \\
\hline Male & 359 & 37.0 \\
\hline \multicolumn{3}{|l|}{ Age } \\
\hline$<25$ & 239 & 24.6 \\
\hline $25-44$ & 473 & 48.8 \\
\hline $45-64$ & 181 & 18.7 \\
\hline$\geqslant 65$ & 77 & 7.9 \\
\hline \multicolumn{3}{|l|}{ Residence } \\
\hline Suburban & 624 & 64.3 \\
\hline Rural & 346 & 35.7 \\
\hline \multicolumn{3}{|l|}{ Education } \\
\hline$>9$ years & 280 & 29.5 \\
\hline$\leqslant 9$ years & 670 & 70.5 \\
\hline Missing & 20 & \\
\hline \multicolumn{3}{|l|}{ Marital status } \\
\hline Single & 275 & 28.5 \\
\hline Married & 512 & 53.1 \\
\hline Divorced & 126 & 13.1 \\
\hline Widowed & 52 & 5.4 \\
\hline Missing & 5 & \\
\hline \multicolumn{3}{|l|}{ Method of suicide } \\
\hline Drugs/other chemicals & 424 & 43.9 \\
\hline Self-cutting & 224 & 23.2 \\
\hline Pesticide & 235 & 24.4 \\
\hline Other violent method & 42 & 4.4 \\
\hline Gas & 28 & 2.9 \\
\hline Hanging & 12 & 1.2 \\
\hline Missing & 5 & \\
\hline
\end{tabular}

year, $3.0 \%$ in the second year and $0.9 \%$ in the fourth year. The timing from the index event to further self-harm ranged from 1 day to 4.7 years (mean 1.2 , s.d. $=1.2$ years).

In the analysis of the predictors of non-fatal repetition as a count outcome, we first looked at the distribution of the count data. The mean number of repetitions in the sample was 1.2 with a standard deviation of 0.2 . The variance was therefore much greater than the mean, suggesting overdispersion of the data. We next ran Poisson and negative binomial regression models, with gender, age, education, marital status and method used at index episode as independent variables. Overall goodness of fit was better for the negative binomial regression model (the likelihood ratio for goodness of fit was 24.09) than for the Poisson regression model (likelihood ratio for goodness-of-fit 39.89). Alpha, the index from the negative binomial model of the extent of overdispersion, was 5.0. The likelihood ratio test for overdispersion results in a chi-squared of $80.20(P<0.001)$ and rejects the null hypothesis of $\alpha=0$. The statistically significant evidence of overdispersion indicates that the negative binomial regression model is preferred to the Poisson regression model.

In the univariate analysis, using negative binomial regression, women and younger people were more likely to repeat self-harm. Regarding the method used in the index episode, those using pesticide were less likely to repeat than others. In the multivariate analysis female gender and method used were independent predictors of repetition (Table 3 ). In a sensitivity analysis we controlled for possible period effects on method used (if, for example, there was a trend towards pesticide use later in the cohort recruitment then those who used pesticide would have a lower risk of repetition simply because there was a shorter follow-up period), by adjusting for follow-up period as a covariate. This did not change the result significantly. 




\section{Discussion}

\section{Strengths and limitations}

To our knowledge, this is the first study from the Far East reporting the non-fatal repetition of self-harm. According to the standard proposed by Owens, ${ }^{1}$ there are some methodological advantages in this study. The sample size is more than 950 cases. A wide range of community services were involved in surveillance in addition to ascertainment from hospital attendance, hence reducing selection bias. The community catchment area was strictly defined. An appropriate statistical paradigm has been used to model factors associated with repetition. The main limitation was that the sample was derived from a register system that provided only limited contextual information, comprising demographic factors and methods of self-harm with no information about other mediators/confounders found in previous studies, such as mental disorders, alcohol misuse, unemployment, physical illness, impulsivity and suicide intent. ${ }^{7,9,10}$ Further studies are needed to address the relationships between these factors and gender as well as suicide methods. Also, it has not been possible for us to validate the completeness of the register system, and under-ascertainment of repeated self-harm cannot be excluded.

\section{Risk of repetition of self-harm}

The cumulative risk of non-fatal repetition is $5.7 \%$ within the first year and $9.5 \%$ over 4 years. The annual risk of repetition is highest within the first year. This result lies well within the wide range of repetition rates reported hitherto, from $1.4 \%$ to $42.0 \%,{ }^{11}$ and close to the report of $9.5 \%$ repeating within a mean follow-up period of 2.8 years, from a study of repeated self-poisoning among 2492 index individuals aged 12 to 20 years. ${ }^{12}$ The repetition rate of self-harm in Nantou was lower than rates reported in a review of findings from Western countries, with a median $16 \%$ (IQR $12-$ $25 \%$ ) by 1 year and $21 \%$ (IQR $12-30 \%$ ) by $1-4$ years. ${ }^{1}$ It was also lower than rates recorded in two large studies from the UK. In a large UK cohort study of 11583 individuals who had self-harmed followed up for an average of 11.4 years (range 1 day to 23 years), $23.0 \%$ repeated self-harm (23.2\% in males; $22.9 \%$ in females). ${ }^{4}$ Another multicentre cohort study with a large sample size $(n=9213)$, carried out during 1997-2002 in north-west England, estimated the incidence of repetition within 12 months to be $13.6 \%{ }^{10}$

There may be methodological explanations for the relatively lower rate of repetition seen in Nantou. First, most (around $80 \%$ ) of the cohort received follow-up consisting of at least one telephone or home visit after the index self-harm episode, carried out by the staff of the Nantou mental health centre. Around $40 \%$ of them accepted detailed assessment and longer follow-up. One previous study showed that even follow-up by simple postcard can reduce the risk of non-fatal repetition. ${ }^{13}$ Since we do not have individual-level data on clinical follow-up, we cannot examine this possibility further. Second, Nantou residents might have moved to other areas, or might have sought help from hospitals outside the district after self-harm episodes. We cannot estimate the size of these effects. Third, although we carefully monitored health services and included other agencies in the surveillance system for the register, we did not formally validate its effectiveness at identifying all self-harm cases. However, real differences may exist between the rates in Asian and Western cultures. A recent study of South Asians in the Manchester area in the UK indicated that they had nearly half the rate of repetition of self-harm compared with White people in the same area ${ }^{14}$ a finding explained either by a true difference in the underlying rate or differences in help-seeking behaviour. A previous study from Iran showed only 


\begin{tabular}{|c|c|c|c|c|c|c|}
\hline Characteristics & $\begin{array}{c}\text { No repetition } \\
n(\%)\end{array}$ & $\begin{array}{l}\text { One or more repetitions } \\
n(\%)\end{array}$ & Crude RR $(95 \% \mathrm{Cl})$ & $P$ & $\begin{array}{l}\text { Adjusted RR } \\
\quad(95 \% \mathrm{Cl})\end{array}$ & $P$ \\
\hline \multicolumn{7}{|l|}{ Gender } \\
\hline Female & $545(89.2)$ & $66(10.8)$ & $2.3(1.4-3.9)$ & 0.002 & $1.9(1.1-3.3)$ & 0.03 \\
\hline Male & 335 (93.3) & $24(6.7)$ & 1 & & 1 & \\
\hline \multicolumn{7}{|l|}{ Age, years } \\
\hline$<25$ & $212(88.7)$ & $27(11.3)$ & 1 & & 1 & \\
\hline $25-44$ & 73 (89.6) & $49(10.4)$ & $1.0(0.6-1.7)$ & 1.00 & $1.1(0.6-1.9)$ & 0.75 \\
\hline $45-64$ & 171 (94.5) & $10(5.2)$ & $0.5(0.2-1.0)$ & 0.06 & $0.7(0.3-1.5)$ & 0.32 \\
\hline$\geqslant 65$ & $424(94.8)$ & $4(5.5)$ & $0.5(0.2-1.5)$ & 0.21 & $0.9(0.3-2.7)$ & 0.88 \\
\hline \multicolumn{7}{|l|}{ Residence } \\
\hline Suburban & $566(90.7)$ & $58(9.3)$ & 1 & & & \\
\hline Rural & $314(90.8)$ & $32(9.2)$ & $1.1(0.7-1.8)$ & 0.69 & & \\
\hline \multicolumn{7}{|l|}{ Education, years $^{\mathrm{C}}$} \\
\hline$\leqslant 9$ & $254(90.7)$ & $26(9.3)$ & $1.1(0.7-1.9)$ & 0.71 & & \\
\hline$>9$ & $610(91.0)$ & $60(9.0)$ & 1 & & & \\
\hline \multicolumn{7}{|l|}{ Marital status ${ }^{\mathrm{c}}$} \\
\hline Single & $243(88.4)$ & $32(11.6)$ & 1 & & & \\
\hline Married & 469 (91.6) & $43(8.4)$ & $0.8(0.5-1.4)$ & 0.48 & & \\
\hline Divorced & $113(89.7)$ & $13(10.3)$ & $1.2(0.6-2.5)$ & 0.58 & & \\
\hline Widowed & $50(96.2)$ & $2(3.8)$ & $0.3(0.1-1.3)$ & 0.10 & & \\
\hline \multicolumn{7}{|l|}{ Method } \\
\hline Pesticides & $224(92.9)$ & $11(4.7)$ & 1 & & 1 & \\
\hline Self-cutting & $203(82.1)$ & $21(9.4)$ & $3.1(1.4-6.7)$ & 0.005 & $2.2(1.0-5.0)$ & 0.05 \\
\hline Drugs or other chemicals & $376(91.7)$ & $48(11.3)$ & $3.2(1.6-6.5)$ & 0.002 & $2.2(1.0-4.6)$ & 0.04 \\
\hline Other violent methods & 39 (95.3) & $3(7.1)$ & $2.3(0.6-8.5)$ & 0.20 & $1.9(0.5-6.9)$ & 0.34 \\
\hline Gas & $23(90.6)$ & $5(17.9)$ & $3.5(0.9-1.4)$ & 0.08 & $3.4(0.9-13.0)$ & 0.08 \\
\hline Hanging & $11(88.7)$ & $1(8.3)$ & $1.6(0 .-18.9)$ & 0.70 & $1.9(0.2-20.1)$ & 0.53 \\
\hline
\end{tabular}

a $4 \%$ incidence of subsequent self-harm in a 3 -year follow-up for 100 self-harm cases. ${ }^{15}$ Another study carried out in Hong Kong regarding the non-fatal repetition of self-harm among Chinese elderly people revealed that the rate of repetition was $3.6 \%$ at follow-up 1.5-4 years after the self-harm, ${ }^{16}$ which was lower than rates reported by Western countries (range 9-18\%). ${ }^{17}$ Another possible explanation emerging from our results is the effect of pesticide use as the method in the index episode of self-harm upon risk of repetition. This method was used by $24 \%$ in the index event, and this group had the least likelihood of repetition. Pesticide is rarely used in acts of self-harm in Western countries. ${ }^{18,19}$

In addition, the effect of earthquake on suicidal behaviour also needs to be considered. Suicide rates were reported to be 1.46 times higher among victims than non-victims in the 2-15 months after the earthquake (95\% CI 1.11-1.92) after adjusting for residential location, age, gender, major disease status, and level of urbanisation. ${ }^{20}$ Another study showed that during the 26 months following the earthquake, the mean monthly suicide rate among the high-exposure group was $42 \%$ higher than the average for the entire observation period. ${ }^{21}$ Thus, it was apparent that the suicide rate increased in the earthquake areas and logically the overall rate of self-harm must have increased too. It may also have inflated the risk of non-fatal self-harm in the present study.

\section{Risk factors of non-fatal repetition of self-harm}

Our finding that females were more likely to repeat the self-harm behaviour was consistent with one previous report from Australia $^{22}$ but differed from the findings from one recent study ${ }^{4}$ in the UK revealing no differences between females and males. This can be partly explained by the association in our data-set between male gender and pesticide use; $41 \%$ of males and $15 \%$ of females used pesticide in the index event, and pesticide use was associated with a lower risk for repetition. However, despite this association, multivariate analysis revealed that gender and pesticide use were independently associated with repetition. The possibility of ascertainment bias is harder to exclude - women might have been no more likely to repeat self-harm, but may be more likely to seek help after doing so because they have been reported to be more likely to seek medical or mental health services than men in a review ${ }^{23}$ and another case-control study. ${ }^{24}$

Our finding of a trend towards higher repetition rates among younger people is consistent with most previous reports. ${ }^{9,25,26}$ However, one study showed that middle-aged people (aged 25-54) had higher repetition rates than the youngest (aged $<25$ ) and the oldest (aged $>54$ ), and the effect of age was mediated by other factors (such as alcohol misuse, psychiatric treatment, unemployment, registered sick and self-injury) in a multivariate model. ${ }^{10}$ Colman et $a l^{7}$ found no association between age and repetition. In our analysis, the effect of age was mediated by choice of method; younger people were less likely to use pesticides for self-harm, which were independently associated with a lower risk of repetition.

The most interesting finding from our study is the low likelihood of self-harm repetition associated with use of pesticide in the index episode. As far as we know, there have been no previous reports of this association. Methods of suicidal behaviour in Asia differ significantly from those employed in Western countries. Firearms are the most frequently used means for suicide in certain Western countries, such as the USA, but these are rarely used in Asia, where poisoning with pesticide is much more frequent. ${ }^{18,19}$ One possible explanation for our findings is that pesticide ingestion is often associated with unpleasant physical 
consequences arising both from the effects of the agent, such as the burning effect on mucosa, pain, tachycardia, vomiting, dizziness, metabolic acidosis, pulmonary oedema, bronchospasm, cyanosis, collapse and coma, and the treatment procedure, including lavage with activated charcoal slurry, intubation, artificial respiration and even tracheotomy. These may act as a deterrent against repetition. In contrast, people using methods such as self-cutting and drugs are more likely to self-harm again. This finding has been reported by one multicentre cohort study in the UK, which demonstrated that self-cutting and self-poisoning with medication had higher repetition rates than other methods. ${ }^{10}$ This reminds us of the necessity to assess these people carefully for risk of repetition despite their choice of methods that are less likely to be lethal.

Vincent C. H. Chen, MD, PhD, Department of Psychiatry and School of Medicine, Chung Shan Medical University, and Department of Psychiatry, Chung Shan Medica Chung Shan Medical University, and Department of Psychiatry, Chung Shan
University Hospital, Taichung, Taiwan; Happy K. L. Tan, MD, Taoyuan Mental University Hospital, Taichung, Taiwan; Happy K. L. Tan, MD, Taoyuan Mental
Hospital, Taoyuan, Taiwan; Andrew T. A. Cheng, MD, PhD, Institute of Biomedica Sciences, Academia Sinica, Taipei, Taiwan; Chung-Ying Chen, MD, Department of Psychiatry, Tsao-Tun Psychiatric Center, Nantou, Taiwan; Long-Ren Liao, Health Bureau, Nantou County Government, Nantou, Taiwan; Robert Stewart, MD, Michael Dewey, PhD, Martin Prince, MD, Section of Epidemiology, Health Services and Population Research Department, Institute of Psychiatry, King's College London, UK

Correspondence: Professor Andrew Cheng, Institute of Biomedical Sciences, Academia Sinica, Taipei, Taiwan, 11529. Email: bmandrew@gate.sinica.edu.tw

First received 6 Apr 2009, final revision 13 Jul 2009, accepted 7 Oct 2009

\section{References}

1 Owens D, Horrocks J, House A. Fatal and non-fatal repetition of self-harm Systematic review. Br J Psychiatry 2002; 181: 193-9.

2 De Leo D, Scocco P, Marietta P, Schmidtke A, Bille-Brahe U, Kerkhof AJ, et al. Physical illness and parasuicide: evidence from the European Parasuicide Study Interview Schedule (EPSIS/WHO-EURO). Int J Psychiatry Med 1999; 29 149-63.

3 Tejedor MC, Diaz A, Castillon JJ, Pericay JM. Attempted suicide: repetition and survival - findings of a follow-up study. Acta Psychiatr Scand 1999; 100 205-11.

4 Zahl DL, Hawton K. Repetition of deliberate self-harm and subsequent suicide risk: long-term follow-up study of 11583 patients. Br J Psychiatry 2004; 185 70-5.

5 Reith DM, Whyte I, Carter G. Repetition risk for adolescent self-poisoning: a multiple event survival analysis. Aust N Z J Psychiatry 2003; 37: 212-8.

6 Evans J, Evans M, Morgan HG, Hayward A, Gunnell D. Crisis card following self-harm: 12-month follow-up of a randomised controlled trial. Br J Psychiatry 2005; 187: 186-7.

7 Colman I, Newman SC, Schopflocher D, Bland RC, Dyck RJ. A multivariate study of predictors of repeat parasuicide. Acta Psychiatr Scand 2004; 109: 306-12.
8 Carter G, Reith DM, Whyte IM, McPherson M. Repeated self-poisoning: increasing severity of self-harm as a predictor of subsequent suicide. Br J Psychiatry 2005; 186: 253-7.

9 Christiansen E, Jensen BF. Risk of repetition of suicide attempt, suicide or all deaths after an episode of attempted suicide: a register-based survival analysis. Aust N Z J Psychiatry 2007; 41: 257-65.

10 Kapur N, Cooper J, King-Hele S, Webb R, Lawlor M, Rodway C, et al. The repetition of suicidal behavior: a multicenter cohort study. J Clin Psychiatry 2006; 67: 1599-609.

11 Sakinofsky I. Repetition of suicidal behaviour. In The International Handbook of Suicide and Attempted Suicide (eds K Hawton, K van Heeringen): 383-404. Wiley, 2000

12 Goldacre M, Hawton K. Repetition of self-poisoning and subsequent death in adolescents who take overdoses. Br J Psychiatry 1985; 146: 395-8.

13 Carter GL, Clover K, Whyte IM, Dawson AH, D'Este C. Postcards from the EDge project: randomised controlled trial of an intervention using postcards to reduce repetition of hospital treated deliberate self poisoning. BMJ 2005; 331: 805 .

14 Cooper J, Husain N, Webb R, Waheed W, Kapur N, Guthrie E, et al. Self-harm in the UK: differences between South Asians and Whites in rates, characteristics, provision of service and repetition. Soc Psychiatry Psychiatr Epidemiol 2006; 41: 782-8.

15 Gharagozlou H, Hadjmohammadi M. Report on a three-year follow-up of 100 cases of suicidal attempts in Shiraz, Iran. Int J Soc Psychiatry 1977; 23: 209-10.

16 Chiu HF, Lam LC, Pang AH, Leung CM, Wong CK. Attempted suicide by Chinese elderly in Hong Kong. Gen Hosp Psychiatry 1996; 18: 444-7.

17 Chan J, Draper B, Banerjee S. Deliberate self-harm in older adults: a review of the literature from 1995 to 2004. Int J Geriatr Psychiatry 2007; 22: 720-32.

18 Beautrais AL. Suicide in Asia. Crisis 2006; 27: 55-7.

19 Cheng ATA, Lee CS. Suicide in Asia and the Far East. In The International Handbook of Suicide and Attempted Suicide (eds K Hawton, $\mathrm{K}$ van Heeringen): 121-35. Wiley, 2000.

20 Chou YJ, Huang N, Lee CH, Tsai SL, Tsay JH, Chen LS, et al. Suicides after the 1999 Taiwan earthquake. Int J Epidemiol 2003; 32: 1007-14.

21 Yang $\mathrm{CH}$, Xirasagar S, Chung HC, Huang YT, Lin HC. Suicide trends following the Taiwan earthquake of 1999: empirical evidence and policy implications. Acta Psychiatr Scand 2005; 112: 442-8.

22 Carter GL, Whyte IM, Ball K, Carter NT, Dawson AH, Carr VJ, et al. Repetition of deliberate self-poisoning in an Australian hospital-treated population. Med J Aust 1999; 170: 307-11.

23 Moller-Leimkuhler AM. The gender gap in suicide and premature death or: why are men so vulnerable? Eur Arch Psychiatry Clin Neurosci 2003; 253 $1-8$.

24 Kung $\mathrm{HC}$, Pearson JL, Liu X. Risk factors for male and female suicide decedents ages 15-64 in the United States. Results from the 1993 National Mortality Followback Survey. Soc Psychiatry Psychiatr Epidemiol 2003; 38: 419-26.

25 Johnsson Fridell E, Ojehagen A, Traskman-Bendz L. A 5-year follow-up study of suicide attempts. Acta Psychiatr Scand 1996; 93: 151-7.

26 Sakinofsky I, Roberts RS. Why parasuicides repeat despite problem resolution. Br J Pychiatry 1990; 156: 399-405. 